\title{
ON THE BOUNDARY OF REDUCED TEICHMÜLLER SPACE ${ }^{1}$
}

\author{
JUDITH C. WASON
}

\begin{abstract}
Quasifuchsian groups used in the description of the boundary of Teichmüller space do not have analogs for reduced Teichmüler space.
\end{abstract}

Let $G$ be a finitely generated nonelementary Fuchsian group of the second kind, with ordinary set $\Omega=\Omega(G)$. In [2] and [3], Earle describes a map of $T^{\#}(G)$, the reduced Teichmüller space of $G$, onto an open bounded domain in a Banach space of bounded quadratic differentials for $G$. This map induces a canonical real analytic structure on $T^{\#}(G)$. Following Bers [1], we discuss the boundary of the image of $T^{\sharp}(G)$.

Earle's map may be described as follows. Choose a cover map $\rho: U \rightarrow \Omega$ satisfying $\rho \circ J(z)=\bar{\rho}(z)$, where $J(z)=-\bar{z}$. (Earle demonstrates in [3] that the analytic structure of $T^{\sharp}(G)$ is actually independent of this choice of cover.) Let $H=\{h \in P S L(2, \mathbf{R}) \mid \rho \circ h=g \circ \rho$ for some $g \in G\}$ be the Fuchsian equivalent of $G . J$ induces a symmetry on $H$, and an anticonformal involution with fixed curves on $U / H$. There is a natural projection $P: H \rightarrow G$, and it is easy to compute that if $P(h)=g$, then $P(\tilde{h})=P(J h J)=g$ also.

Define $M_{1}(H)\left(M_{1}(G)\right)$ as the open unit ball in the space of Beltrami differentials of $H(G)$. Set $B_{2}(H, L)$ as the set of bounded quadratic differentials of $H$ $\left(\|\phi\|=\sup \left|\phi(z)(z-\bar{z})^{2}\right|<\infty\right)$. Let $B_{2}(G, \Omega)$ be the set of bounded quadratic differentials for $G$, real on $\mathbf{R} \cap \Omega\left(\|\phi\|=\sup \left|\phi(z) \lambda^{-2}\right|<\infty\right.$ where $\lambda$ is the Poincaré metric on $\Omega$ induced by $\rho)$.

Let $M_{1}^{\prime}(H)=\left\{\mu \in M_{1}(H) \mid \mu(J(z))=\bar{\mu}(z)\right\}$ and $B_{2}^{\prime}(H, U)=\{\phi \in$ $\left.B_{2}(H, U) \mid \phi(J(z))=\bar{\phi}(z)\right\}$. Then there are natural isomorphisms $M_{1}(G) \rightarrow M_{1}^{\prime}(H)$ given by $\mu \rightarrow \rho \cdot \mu=\mu(\rho(z)) \overline{\rho^{\prime}(z)} / \rho^{\prime}(z)$ and $B_{2}(G, \Omega) \rightarrow B_{2}^{\prime}(H, L)$ given by $\phi \rightarrow \phi$ $\times \rho$ where $\overline{\phi \times \rho(\bar{z})}=\phi(\rho(z)) \rho^{\prime}(z)^{2}$.

Let $w_{\mu}$ be the unique normalized solution of the Beltrami differential equation $w_{\bar{z}}=\mu w_{z}$ on $\bar{C}$, where $\mu$ has been extended to $L$ by $\mu(\bar{z})=\bar{\mu}(z), \mu \in M_{1}(H)$ or $M_{1}(G)$. Then $T(H)\left(T^{\sharp}(G)\right)$ the reduced Teichmüller space is defined as the set of equivalence classes $\left[w_{\mu}\right]$, where $w_{\mu} \sim w_{\nu}(\mu \sim \nu)$ if and only if $w_{\mu}=w_{\nu}$ on the limit set $\Lambda(H)$ of $H(\Lambda(G)$ of $G) . T^{\prime}(H)$ is the set of $\left[w_{\mu}\right]$ which are odd on R. Note that $\mu \in M_{1}^{\prime}(H)$ if and only if $w_{\mu} \circ J=J \circ w_{\mu}[2]$, and each $\left[w_{\mu}\right] \in T^{\prime}(H)$ contains at

Received by the editors December 26, 1978 and, in revised form, June 7, 1979.

AMS (MOS) subject classifications (1970). Primary 32G15.

Key words and phrases. Teichmüller space, reduced Teichmüller space, Beltrami differential, quadratic differential, quasifuchsian group, Schwarzian.

'This research was conducted while the author was a visitor at Harvard University. 
least one such $w_{\mu}$, [5]. Let $w^{\mu}$ be the normalized solution when $\mu$ is set equal to zero on $L$.

Then the following map of $T^{\sharp}(G)$ into $B_{2}(G, \Omega)$ is well defined

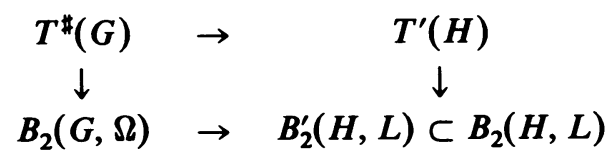

by

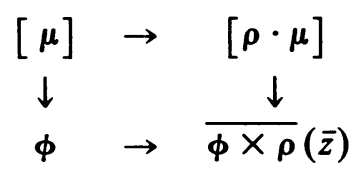

where $\overline{\phi \times \rho}(\bar{z})=\left\{w^{\rho \cdot \mu}, z\right\}$, the Schwarzian derivative of $w^{\rho \cdot \mu}$, and horizontal arrows represent real analytic isomorphisms. $T^{\prime}$ and $T^{\#}$ will be identified with their images. (Recall that for groups of the second kind, $[\mu] \rightarrow\left\{w^{\mu}, z\right\}$ is not well defined.)

We will describe how the symmetric structure of $H$ is reflected in the cusps on the boundary of $T^{\prime}(H) \subset T(H)$, and then show that this description cannot be applied to the group $G$, even through the relationship between $T^{\prime}(H)$ and $T^{\sharp}(G)$ is so close.

As in [1], we form two classes of groups. If $\phi \in B_{2}(H, L) \cap T(H)$, there exists a quasiconformal map $w_{\phi}: C \rightarrow C$, which satisfies the differential equation $w_{\bar{z}}=\mu w_{z}$, $\mu \in M_{1}(H), \mu=0$ on $L$, and $\left\{w_{\phi}, z\right\}=\phi$. By Theorems 1 and 2 of [2], $T^{\prime}=T \cap$ $B^{\prime}$; in particular, each $\phi \in T^{\prime}(H)$ is the Schwarzian derivative of a quasiconformal map $w_{\phi}$ with $\mu \in M_{1}^{\prime}(H)$. It is clear that if a sequence $\left\{\phi_{n}\right\} \in B_{2}^{\prime}(H, L)$ has limit $\phi_{\infty}$, then $\phi_{\infty} \in B_{2}^{\prime}(H, L)$ also. An isomorphism $\chi_{\phi}$ is defined for $h \in H$ by $w_{\phi}(h(z))=\chi_{\phi}(h) \circ w_{\phi}(z)$. The isomorphism $\chi_{\phi}(H)$ is the first group.

The second class of groups is defined as follows. The quasifuchsian group $\chi_{\phi}(H)$, $\phi \in T(H)$, represents two Fuchsian groups obtained by conjugating $\chi_{\phi}(H)$ by conformal maps of $w_{\phi}(U)$ and $w_{\phi}(L)$ onto $U$ and $L$ respectively. For the lower map, send $w_{\phi}(0), w_{\phi}(1)$ and $w_{\phi}(\infty)$ onto $0,1, \infty$ respectively; then the lower image is $H$. We normalize the map $\omega: w_{\phi}(U) \rightarrow U$ so that $\omega \circ w$ fixes $0,1, \infty$; then $\hat{\chi}_{\phi}(H)=H_{\mu}=(\omega \circ w) \circ H \circ(\omega \circ w)^{-1} ; \hat{\chi}_{\phi}$ depends only on $\phi$, and not on the choice of $\mu$. Note that $H_{\mu}$ is precisely the group obtained by conjugating $H$ by the unique solution $w_{\mu}$ of the differential equation $w_{\bar{z}}=\mu w_{z}$; mapping $U \rightarrow U$, fixing 0 , $1, \infty$.

LEMMA 1. Let the closed curve $C$ on $U / H$ determine the conjugacy class of the element $h$ of $H$. Then $\tilde{C}$, the curve symmetric to $C$, determines the conjugacy class of the element $\tilde{h}=J \circ h \circ J$ of $H$.

We note that $C$ and $\tilde{C}$ are freely homotopic if and only if they are also freely homotopic to a boundary curve of $U / G$; in this case all three will determine conjugate elements of $\boldsymbol{H}$. 
LEMMA 2. Suppose $h \in H$ is such that $\lim _{j \rightarrow \infty}\left(\operatorname{trace} \hat{\chi}_{\phi}(h)\right)^{2}=4, \phi_{j} \in T^{\prime}(H)$. Then $\lim _{j \rightarrow \infty}\left(\text { trace } \hat{\chi}_{\phi}(\tilde{h})\right)^{2}=4$ also.

Proof. Since each $\phi_{j} \in T^{\prime}(H)$, we may choose a representative $w_{\phi}$ where $w_{\phi}$ has dilatation $\mu$ on $U, \mu \in M_{1}^{\prime}(H)$. Then $w_{\mu}$ commutes with $J$, and

$$
\begin{aligned}
\hat{\chi}_{\phi}(\tilde{h}) & =w_{\mu} \circ \tilde{h} \circ w_{\mu}^{-1}=w_{\mu} \circ J \circ h \circ J \circ w_{\mu}^{-1} \\
& =J \circ w_{\mu} \circ h \circ w_{\mu}^{-1} \circ J=J \circ \hat{\chi}_{\phi}(h) \circ J .
\end{aligned}
$$

Hence $\left(\operatorname{trace} \hat{\chi}_{\phi}(h)\right)^{2}=\left(\operatorname{trace} \hat{\chi}_{\phi}(\tilde{h})\right)^{2}$ for each $j$.

From Lemma 1 we conclude that "pinching" one loop via a $\phi, \phi \in T^{\prime}(H)$, induces a "pinching" of the symmetric loop. We next demonstrate the existence of appropriate $\phi_{j}$ 's.

Proposition. Let $\hat{C}=\left\{C_{1}, C_{2}, \ldots, C_{N}\right\}$ be a set of disjoint simple loops on $U / H$, no two of which are freely homotopic, and such that if $C_{k} \in C$, then the curve symmetric to $C_{k}, \tilde{C}_{k} \in \hat{C}$. Let the elements $h_{k}$ determined by the $C_{k}$ be hyperbolic. Then there is a mapping $t \rightarrow \phi_{t}$ of $0<t<\infty$ into $T^{\prime}(H)$ such that

$$
\lim _{t \rightarrow \infty}\left(\operatorname{trace} \hat{\chi}_{\phi_{t}}\left(h_{k}\right)\right)^{2}=\lim _{t \rightarrow \infty}\left(\operatorname{trace} \hat{\chi}_{\phi_{1}}\left(\tilde{h}_{k}\right)\right)^{2}=4, \quad k=1, \ldots, N
$$

hence there are cusps on the boundary of $T^{\prime}(H)$.

Proof. We assume that $\hat{C}$ contains one pair of symmetric loops, $C$ and $\tilde{C}$, and leave the general case to the reader. Suppose $C$ is not homotopic to a curve fixed by the anticonformal involution of $U / H$. Then $C$ is distinct from and not homotopic to $\tilde{C}$. Follow the construction of Bers [1, Theorem 11]. Replace an annulus $D, 0<a<|\xi|<1$ with $C$ homotopic to $|\xi|=\sqrt{a}$, by the annulus $D_{t}$, $0<a^{t}<|\xi|<1$. Similarly replace the annulus $\tilde{D}$ symmetric to $D$, by $D_{t}$, symmetric to $D_{t}$. If $C$ is homotopic to a boundary curve, it is also homotopic to $\tilde{C}$; hence $C=\tilde{C}$. Then $C$ may be bordered by two symmetric annuli $A_{1}$ and $A_{2}, 0<a<|\xi|$ $<1$ and $0<a<\left|\xi^{\prime}\right|<1$, respectively. Let $D=A_{1} \cup A_{2}$ identified along the curves $|\xi|=\left|\xi^{\prime}\right|=a$. We may replace the annulus $D$ by the annulus $D_{t}$ obtained by replacing $A_{i}$ by the symmetric annuli $A_{i t}, 0<a^{t}<|\xi|<1, i=1,2$, again properly identified along the boundaries. Then the module of $D_{t}$ is equal to twice the module of $A_{i t}$, and hence becomes infinite as $t$ goes to infinity.

We let $S^{(t)}$ be the symmetric surface obtained by replacing each $D$ by the corresponding $D_{t}$. Then there is a map $f_{t}: U / H=S \rightarrow S^{(t)}$, respecting the symmetries, which is the identity on $S-\{D\}$ and maps a point $r e^{i \theta}$ of $D\left(A_{i}\right)$ onto $r^{t} e^{i \theta}$ of $D_{t}\left(A_{i t}\right)$. The anticonformal involution $\sigma_{t}$ on $S^{(t)}$ lifts to $U$; replacing the universal cover $\pi$ by $\pi \circ A, A \in P S L(2, \mathbf{R})$, if necessary, we may assume this lift is $J$, [2]. Hence we may lift $f_{t}$ to a map $w_{t}: U \rightarrow U$ such that $w_{t} \circ J=J \circ w_{t}$, and the group $H_{t}=w_{t} \circ H \circ w_{t}^{-1}$ is a Fuchsian group representing the symmetric surface $S^{(t)}=$ $U / H_{t}$.

For each $t$, there is a $\phi_{t} \in T(H)$ such that $\hat{\chi}_{\phi_{1}}(h)=w_{t} \circ h \circ w_{t}^{-1}$. If $w_{t}=w_{\mu}$ commutes with $J,\left\{w^{\mu}, z\right\}=\phi \in B_{2}^{\prime}(H, U)$, and so $\phi_{t}$ is actually in $T^{\prime}(H)$. 
The rest of the proof follows as in Theorems 11 and 12 of [1], since the moduli of the various $D_{t}$ become infinite, and the limit of a sequence of elements of $T^{\prime}(H)$ is also symmetric.

We next attempt to determine how the relationships of $H, \chi(H)$ and $\hat{\chi}(H)$ apply to the group $G$.

For $\mu \in M_{1}(G), w_{\mu}$ satisfies $w_{\mu}(\bar{z})=\overline{w_{\mu}(z)}$. Then the normalized mapping $w_{\rho-\mu}$ has the property that $w_{\mu} \circ \rho \circ\left(w_{\rho \cdot \mu}\right)^{-1}=\rho_{\mu}$ is a holomorphic cover map $U \rightarrow$ $\Omega\left(G_{\mu}\right)$. Now $\rho_{\mu} \circ J=\bar{\rho}_{\mu}$, and $H_{\rho \cdot \mu}$ is the Fuchsian equivalent of $G_{\mu}$. Further, since $w_{\rho \cdot \mu}$ commutes with $J$, both $w_{\rho \cdot \mu} \circ h \circ\left(w_{\rho \cdot \mu}\right)^{-1}$ and $w_{\rho \cdot \mu} \circ \tilde{h} \circ\left(w_{\rho-\mu}\right)^{-1}=$ $J \circ w_{\rho-\mu} \circ h \circ\left(w_{\rho-\mu}\right)^{-1} \circ J$ will project to a given $g \in G_{\mu}$.

ThEOREM. Any quasifuchsian analog of $\chi(H)$ is actually Fuchsian.

Proof. Suppose we have defined some quasifuchsian group $\chi_{\phi}(G)$, such that $\chi_{\phi \times \rho}(H)=\left\{\gamma \mid \eta \circ \pi=\pi \circ \gamma\right.$ for some $\left.\eta \in \chi_{\phi}(G)\right\}$ where $\pi$ is a cover map from the simply connected region $w^{\rho \cdot \mu}(L)$ onto $\Omega\left(\chi_{\phi}(G)\right)$. By the definitions of $H$ and $H_{\rho \cdot \mu}=\hat{\chi}_{\phi \times \rho}(H)$, the elements $h$ and $\tilde{h}=J h J$ are paired in the projection to $G$, and $h_{\rho \cdot \mu}$ and $\tilde{h}_{\rho \cdot \mu}$ are paired in the projection to $G_{\mu}$. Hence for a well-defined $\chi_{\phi}(G)$, $h^{\mu}=\chi_{\phi \times \rho}(h)$ and $\tilde{h}^{\mu}=\chi_{\phi \times \rho}(\tilde{h})$ should project to one element of $\chi_{\phi}(G)$. Since $T^{\prime}=T \cap B^{\prime}$ and $\left\{w^{\rho \cdot \mu}, z\right\} \in B^{\prime}(H, L)$ then $w^{\rho \cdot \mu} \circ J \circ\left(w^{\rho \cdot \mu}\right)^{-1}=\bar{K}$ is the conjugate of an involutory fractional linear transformation on $w^{\rho \cdot \mu}(L)$, [2]. But then

$$
\begin{aligned}
\chi(\tilde{h}) & =w^{\rho \cdot \mu} \circ J \circ h \circ J \circ\left(w^{\rho \cdot \mu}\right)^{-1} \\
& =\bar{K} \circ w^{\rho \cdot \mu} \circ h \circ\left(w^{\rho \cdot \mu}\right)^{-1} \circ(\bar{K})^{-1}=\bar{K} \chi(h)(\bar{K})^{-1}
\end{aligned}
$$

and $\bar{K}$ induces a well-defined automorphism of any subgroup of $\chi(H)$ containing both elements to be paired, in particular, of the cover group of $\pi$. Then $\bar{K}$ must project to an anticonformal involution $\bar{k}$ of $\Omega\left(\chi_{\phi}(G)\right)$. But if $g \circ \pi=\pi \circ \chi(h)=$ $\pi \circ \chi(\tilde{h})$, then

$\bar{k} \circ g \circ \pi=\bar{k} \circ \pi(\chi(\gamma))=\pi \circ \bar{K} \circ \chi(\gamma)=\pi \circ \chi(\tilde{\gamma}) \circ \bar{K}=g \circ \pi \circ \bar{K}=g \circ \bar{k} \circ \pi$, and since $\pi$ is a cover map, $\bar{k} \circ g=g \circ \bar{k}$ on $\Omega\left(\chi_{\phi}(G)\right)$. But then by [4], $\bar{k}$ is the restriction of an anticonformal involutory fractional linear transformation, and $\chi_{\phi}(G)$ is in fact Fuchsian.

\section{REFERENCES}

1. L. Bers, On boundaries of Teichmïller spaces and on Kleinian groups. I, Ann. of Math. (2) 91 (1970), $570-600$.

2. C. J. Earle, Teichmiiller spaces of groups of the second kind, Acta Math. 112 (1964), 91-97.

3. __ Rechuced Teichmïller space, Trans. Amer. Math. Soc. 126 (1967), 54-63.

4. I. Kra and B. Maskit, Inoolutions on Kleinian groups, Bull. Amer. Math. Soc. 78 (1972), 801-805.

5. J. C. Wason, On the straightness of reduced Teichminller space, Proc. Amer. Math. Soc. 56 (1970), 193-198.

Departient of Mathematics, Wellesley College, Wellesley, Massachusetts 02181

Current address: Rural Route 1, Box 155, Tuxedo Park, New York, 10987 